\title{
Simulador de crecimiento y secuestro de carbono para plantaciones de teca en Venezuela: una aplicación en SIMILE
}

\author{
Growth and carbon sequestration simulator for teak plantations in Venezuela: \\ An application in SIMILE
}

\author{
Mauricio Jerez ${ }^{\text {a*, }}$, María Quintero ${ }^{\text {b }}$, Ana Quevedo ${ }^{\text {, }}$, Ana Moret ${ }^{\text {b }}$

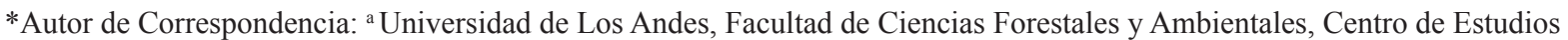 \\ Forestales de Postgrado, Vía Principal Chorros de Milla, Mérida, Venezuela, tel.: 542748481169, jerezorama@gmail.com \\ ${ }^{\mathrm{b}}$ Universidad de Los Andes, Facultad de Ciencias Forestales y Ambientales, Escuela de Ingeniería Forestal, Mérida, Venezuela. \\ c Universidad de Los Andes, Facultad de Ciencias Forestales y Ambientales, Escuela Técnica Superior Forestal, Mérida, Venezuela.
}

\section{SUMMARY}

Simulation is a highly valuable tool for environmental, ecological and forestry research, and in decision making for environmental resources management. Simulation models allow representing the complexity of various processes and integrating the information from many investigations in a system that can be used to predict its future state and to evaluate the effect of decision alternatives. The aim of this work was to illustrate the potential of the declarative-visual programming languages for representing, implementing and simulating in a simple way a relatively complex forest system. A growth and yield simulator prototype was created for teak plantations (Tectona grandis). The model consists of a differential equations system following the space-state approach. The simulator was implemented in SIMILE v.6.0, a declarative-visual language, and was used to analyze plantation growth, harvestable volume and carbon sequestration capacity in response to silvicultural management scenarios: site quality, initial spacing and thinning schedule. This work is an example of the potential of these languages, particularly for educational purposes because it facilitates the experimentation of alternatives and sensitivity analyses by simultaneously testing many scenarios to assess the effect of changes in model parameters, to examine model assumptions, data quality, construction approach, computing algorithm efficiency and predictions quality.

Key words: growth, basal area and yield models, Tectona grandis, state-space approach.

\section{RESUMEN}

La simulación es una herramienta de gran valor en la investigación ambiental, ecológica y forestal; como también en los procesos de toma de decisiones para el manejo de recursos ambientales. Los modelos de simulación permiten representar la complejidad de diversos procesos e integrar la información de numerosas investigaciones en un sistema que puede utilizarse para predecir su estado futuro y evaluar el efecto de alternativas de decisión. El objetivo de este trabajo fue ilustrar el potencial de los lenguajes declarativos visuales para representar, implementar y simular de manera sencilla un sistema forestal relativamente complejo. Se creó un prototipo de simulador del crecimiento y rendimiento para plantaciones de teca (Tectona grandis). El modelo se basó en un sistema de ecuaciones diferenciales de acuerdo al enfoque de espacio de estados. El simulador se implementó en SIMILE v. 6.0 un lenguaje declarativo-visual y fue utilizado para analizar el crecimiento de las plantaciones, su volumen aprovechable, y la capacidad de secuestro de carbono en respuesta a escenarios de manejo silvicultural: calidad de sitio, espaciamiento inicial y régimen de aclareo. Este trabajo es un ejemplo del potencial de estos lenguajes, particularmente para propósitos educativos, al facilitar la experimentación de alternativas y análisis de sensibilidad al poder analizarse muchos escenarios simultáneamente, examinar el efecto de cambios en los parámetros del modelo, el examen de los supuestos, la calidad de los datos, el enfoque de construcción, la eficiencia de los algoritmos de computación y la calidad de las predicciones.

Palabras clave: modelos de crecimiento y rendimiento, área basal, Tectona grandis, espacio de estados.

\section{INTRODUCCIÓN}

La simulación es una herramienta de gran valor en la investigación ambiental, ecológica y forestal; así como en los procesos de toma de decisiones en el manejo de recursos ambientales. Los modelos permiten representar la complejidad de diversos procesos e integrar la información de nu- merosas investigaciones en un único sistema que puede ser utilizado para predecir el estado futuro y evaluar los efectos de diferentes alternativas de decisión. Comparando las predicciones con datos reales, podemos contrastar nuestra comprensión del sistema. Analizando la respuesta del sistema al cambiar las entradas al mismo, podemos explorar el impacto de factores externos (ejemplo: cambio climático) 
y acciones de manejo (ejemplo: reducción de emisiones de carbono) (Muetzelfeldt y Taylor 2004). En el campo forestal, los modelos de simulación del crecimiento y rendimiento, han alcanzado un enorme desarrollo y hoy día son muy importantes no sólo para tomar decisiones en el manejo de bosques naturales y plantaciones forestales, sino también para guiar las políticas forestales en muchos países.

En la investigación forestal se distingue entre modelos para predecir y modelos para comprender (García 1988, Weiskittel et al. 2011). Los modelos predictivos también llamados modelos de crecimiento y rendimiento, los cuales según el nivel de resolución se clasifican en modelos de rodal, modelos de clase de tamaño y los modelos de base individual. Los modelos de rodal son construidos utilizando variables que describen el desarrollo del rodal, requiriendo relativamente poca información para simular el crecimiento y desarrollo del bosque, y son muy útiles en el modelado de plantaciones forestales (Peng 2000).

El enfoque de espacio de estados permite modelar el crecimiento de un rodal mediante un sistema de ecuaciones diferenciales que representan la dinámica de unas pocas variables (ejemplo: altura, área basal y densidad) que describen el estado del sistema en un momento dado (incluyendo las condiciones iniciales). Se asume que las variables de estado resumen los eventos que afectarán el desarrollo del rodal, por lo tanto, los estados futuros pueden ser determinados por el estado presente y las acciones futuras (silvicultura), mientras que otras variables de interés (ejemplo: volumen total, comercial, contenido de carbono) pueden derivarse a partir de las variables de estado (García 2013). La formulación general de un modelo bajo este enfoque ha sido descrita por García $(1994,2013)$ y Quintero et al. (2012).

Actualmente, la mayoría de los modelos son implementados como programas escritos en lenguajes de programación convencional tales como $\mathrm{C}++$. La visión predominante en la comunidad científica es que el papel de las computadoras en el proceso de modelado es únicamente hacer los cálculos necesarios para simular el comportamiento del sistema modelado, es decir, se tiene una visión de procedimiento consistente en desarrollar el programa para que ejecute una serie de instrucciones. Este enfoque ha presentado muchos obstáculos en el aprovechamiento de todo el potencial que la simulación puede ofrecer para investigación y aplicaciones, particularmente con el enorme desarrollo y versatilidad de las computadoras. Entre los problemas que comúnmente enfrentan los modeladores está el costo en tiempo necesario para construir, documentar e implementar un modelo, y particularmente en desarrollar una buena interfaz con el usuario. Igualmente, es difícil entender los modelos desarrollados por otros y compartir los componentes de distintos modelos debido a diferencias en los enfoques y lenguajes de programación.

En la última década ha tomado impulso el enfoque denominado modelado declarativo, basado en el principio de la representación del modelo en la computadora como una especificación de su estructura conceptual y matemática, en lugar de instrucciones que indican al computador la ejecución de una serie de cálculos. La especificación define los objetos y variables en el modelo, así como las relaciones entre ellos. Esto permite tratar el modelado como un proceso de diseño en el cual los modeladores deciden qué componentes y variables incluir en sus modelos y como deben conectarse (Muetzelfeltd y Taylor 2004). Para ello, se han desarrollado herramientas computacionales que facilitan el proceso de construir y trabajar con modelos, similares a las herramientas CAD (Computer-Aided Design) existentes en disciplinas como la arquitectura y la electrónica para ayudar en el diseño de edificios y circuitos electrónicos. Una característica clave de este enfoque es la separación entre la representación del diseño y las herramientas empleadas para procesar el mismo. Por otra parte, las interfaces gráficas (graphical user interfaces -GUIs-) han revolucionado la facilidad con la cual gente poco especializada en computación puede acceder a recursos computacionales complejos. En el campo de la simulación se han desarrollado los llamados lenguajes de programación visual que permiten construir modelos sin necesidad de manejar lenguajes de programación convencionales o la sintaxis de un lenguaje de simulación.

El lenguaje declarativo-visual SIMILE fue diseñado originalmente para modelar y simular sistemas agroforestales (Muetzelfeldt y Taylor 2001), pero es muy útil también para modelar sistemas ecológicos y forestales (Vanclay 2003). SIMILE posee una interfaz gráfica muy intuitiva que permite construir fácilmente modelos complejos sin grandes habilidades de programación (Vanclay 2003). Los ambientes de modelado visual, basados en el paradigma de dinámica de sistemas, han facilitado enormemente el acceso al campo de la simulación a estudiantes e investigadores con pocas habilidades matemáticas y de programación (Muetzelfeldt y Massheder 2003).

El objetivo de este trabajo es desarrollar e implementar, mediante SIMILE, un simulador del crecimiento, rendimiento volumétrico maderable y almacenamiento de carbono para plantaciones de teca (Tectona grandis Linn. F.) y su respuesta a escenarios de manejo silvicultural, incluyendo calidad de sitio, espaciamiento inicial y régimen de aclareo a fin de ilustrar el potencial de los lenguajes de modelado visual declarativo en aplicaciones forestales.

\section{MÉTODOS}

Se utilizó el lenguaje SIMILE para diseñar e implementar el simulador. De acuerdo con la filosofía de este lenguaje, primero, se dibujaron los diagramas que muestran las principales características del modelo (diseño); segundo, se agregó información cuantitativa (valores y ecuaciones) a los elementos del diagrama. El modelo se representó como un conjunto de declaraciones que definen su estructura del modelo, de manera similar a la verbal. Estas declaraciones se almacenaron en un archivo de texto (no en código binario), lo cual da gran flexibilidad de com- 
partir modelos entre diferentes ambientes de modelado. La notación en SIMILE se basa en la notación estándar de dinámica de sistemas (Forrester 1961) usada en muchos lenguajes de modelado (ejemplo: Powersim, Stella, Vensim), siendo, por tanto, accesible a otros investigadores, incluso no familiarizados con SIMILE. Esta notación es intuitiva y ampliamente usada en varias disciplinas para describir sistemas continuos, ya que se basa en diagramas que combinan los conceptos de cantidad, flujo e influencias. Se emplearon cuatro símbolos básicos para representar un sistema dinámico: 1) el compartimiento $\square$ (depósito, nivel, o variable de estado) representa cantidades, por ejemplo, carbono, alturas, diámetros; 2) el flujo 륨 representa un proceso (ecuación diferencial) que contribuye a la tasa de cambio de un compartimiento. Un compartimiento puede tener múltiples flujos de entrada y de salida; 3) las variables representan otras cantidades en el sistema tales como parámetros, variables intermedias, variables exógenas o variables de salida, y la expresión matemática para calcular su valor; y 4) la flecha de influencia $r$, representa las relaciones entre los diferentes elementos del modelo. Por ejemplo, el crecimiento en volumen de un árbol (V) puede describirse como una variable de salida que depende de dos variables de estado, la altura (h) y el área basal (g), definida mediante el siguiente sistema de ecuaciones [1]:

$$
\frac{d h}{d t}=a_{1} h^{b_{1}}-c_{1} h \quad \text { y } \frac{d g}{d t}=a_{2} g^{b_{2}}-c_{2} g
$$

donde,

$d h / d t$ y $d g / d t$ son las tasas de cambio para la altura y área basal con el tiempo respectivamente. $a_{\mathrm{i}}, b_{\mathrm{i}}$ y $c_{\mathrm{i}}$ son constantes (figura 1).

Diseño del modelo. De manera similar a [1], en [2] las condiciones de un rodal en cualquier tiempo $t$ fueron definidas por las variables de estado: altura mayor $(H, \mathrm{~m})$, área basal $\left(G, \mathrm{~m}^{2} \mathrm{ha}^{-1}\right)$ y densidad ( $N$, número de árboles por hectárea). El sistema de ecuaciones diferenciales conformado por funciones de transición, una para cada variable de estado, permitió proyectar el estado futuro del rodal. A partir de las variables de estado se calculó el volumen maderable y el secuestro de carbono como principales variables de salida.

$$
\frac{d H}{d t}=f_{1}(H) ; \frac{d G}{d t}=f_{2}(G) ; \frac{d N}{d t}=f_{3}(G)
$$

El crecimiento en altura mayor se modeló mediante una ecuación de Chapman-Richards [3].

$$
\frac{d H}{d t}=\beta_{1} H^{\beta_{2}}-\beta_{3} H
$$

Los parámetros $\beta_{1} \beta_{2}, \beta_{3}$ fueron estimados mediante el método de la diferencia algebraica (Quintero et al. 2012), generándose una familia de curvas anamórficas de índice de sitio (edad base = 16 años) a partir de la curva guía, que representa la tendencia promedio de crecimiento en altura mayor. Se diferenciaron tres clases de sitio: clases I, II y III, con índices de sitio 24, 21 y 18 respectivamente a la edad base de 16 años. La altura promedio del rodal (ecuación [4]) se estimó como una función de la altura mayor y de la densidad del rodal.

$$
H_{p}=\delta \cdot H \cdot C_{c}
$$

Donde: $H_{p}=$ altura promedio (m), $H=$ altura mayor (m), $\delta=$ parámetro a estimar, $C_{c}=$ razón árboles removidos respecto a los árboles remanentes.

En rodales no aclareados existe una alta correlación entre estas variables, la cual puede alterarse dependiendo de la clase de aclareo que se realice. Por ejemplo, en un
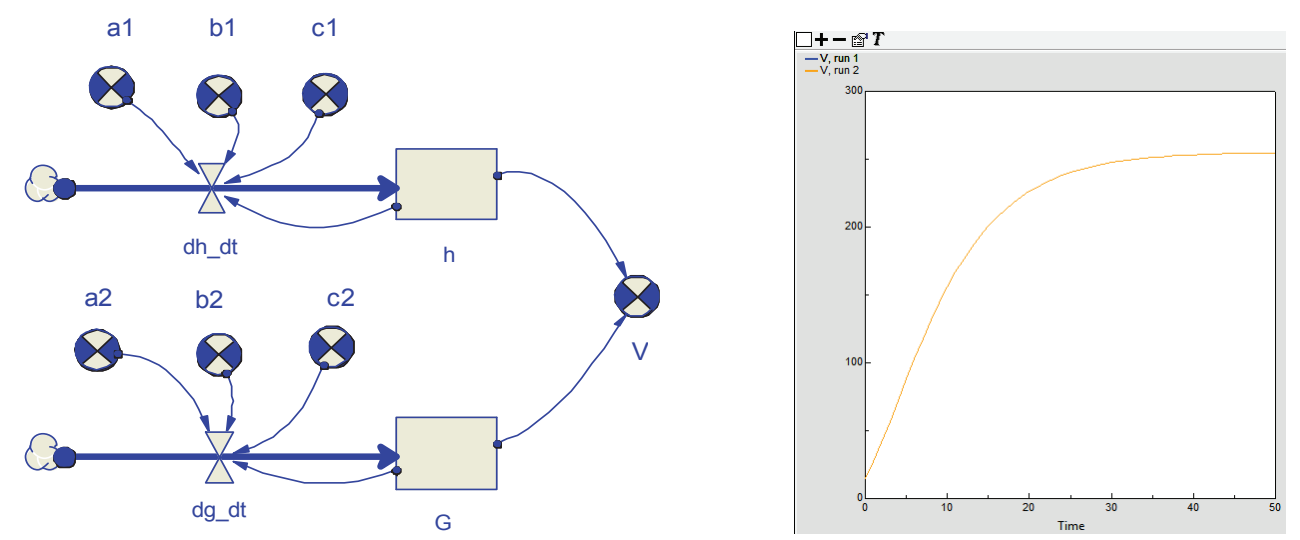

Figura 1. Representación del crecimiento en volumen de un árbol como un sistema dinámico empleando la notación de Forrester.

Representing the volume growth of a tree as a dynamical system using the Forrester's notation. 
aclareo donde se eliminen los árboles más pequeños, la altura promedio tenderá a aproximarse a la altura de los árboles dominantes.

Para describir el crecimiento en área basal se utilizó el modelo de Chapman-Richards ajustado por Zambrano et al. (1995) para plantaciones de teca. La forma integral de la ecuación [5] es:

$$
G_{t}=G_{p s} \cdot\left[1-e^{-\beta_{4}\left(t-t_{o}\right)}\right]^{\frac{1}{1-\beta_{5}}}
$$

Donde,

$G_{t}=$ área basal al tiempo $\mathrm{t}\left(\mathrm{m}^{2} \mathrm{ha}^{-1}\right) . G_{p s}=$ área basal potencial de sitio $\left(\mathrm{m}^{2} \mathrm{ha}^{-1}\right), t_{0}=$ tiempo inicial de medición del área basal (años). $t=$ edad (años). $\beta_{4}=$ parámetro que se relaciona con la tasa de crecimiento. $\beta_{5=}$ parámetro relacionado con el punto de inflexión de la curva.

La forma diferencial de [5] es [6],

$$
\frac{d G}{d t}=\beta_{4} \cdot G_{t}\left[\frac{\left(G_{p s} / G_{t}\right)^{1-\beta_{5}}-1}{1-\beta_{5}}\right]
$$

Donde, $d G / d t=$ incremento en área basal respecto al tiempo y los demás símbolos como en [5].

El área basal potencial del sitio $\left(G_{p s}\right)$ se refiere al área basal máxima que una especie o tipo de vegetación puede alcanzar, dependiendo de las condiciones del sitio (ej. fertilidad, precipitación). Para la teca, en calidades de sitio muy buenas, $G_{p s}$ se aproxima a $37,5 \mathrm{~m}^{2} \mathrm{ha}^{-1}$ (Zambrano et al. 1995, Bermejo et al. 2004, Pérez y Kanninen 2005). El índice de sitio se correlaciona con el área basal potencial de sitio, de manera que a menor altura mayor $(H)$, esta también es menor. Para las calidades de sitio I, II y III, el área basal potencial de sitio es 37,$5 ; 34$; y $30 \mathrm{~m}^{2} \mathrm{ha}^{-1}$, respectivamente.

Para simular cambios en la tasa de crecimiento del rodal después de un aclareo, el modelo modifica automáticamente el valor de $\beta_{4}$ en ecuación [6], hasta un máximo de cuatro aclareos (Pienaar y Turnbull 1973, Zambrano et al. 1995). Asumiendo una densidad constante, y dado el valor de $G_{p s}$, el coeficiente $\beta_{5}$, la edad inicial $\left(t_{0}\right)$ y la edad actual $(t)$, el valor de $\beta_{4}$ que determina la trayectoria del crecimiento en área basal posterior a un aclareo viene dada por la ecuación [7]:

$$
\beta_{4}=\frac{\left|\ln \left[1-\left(\frac{G_{t}}{G_{p s}}\right)^{1-\beta_{5}}\right]\right|}{t-t_{0}}
$$

El comportamiento de $\beta_{4}$ fue estimado por Zambrano et al. (1995) para plantaciones de teca con diferentes espaciamientos iniciales y número e intensidad de aclareos. El modelo permite representar los efectos del régimen de aclareo aplicado a una plantación. Los cambios instantáneos en diámetro cuadrático del rodal posteriores a la realización de un aclareo se determinaron relacionando el diámetro, densidad y área basal de los vuelos original, extraído y remanente mediante [8] y [9]:

$$
\begin{gathered}
d_{d}=\sqrt{\left(1+\frac{N_{e}}{N_{d}}\right) \cdot\left(1-\frac{d_{e}}{d_{a}}\right)^{2} \cdot d_{a}} \\
N_{e}=\left(1-\left(1-\frac{G_{e}}{G}\right)^{1 / c_{c}}\right)
\end{gathered}
$$

Donde,

$d_{d}=$ diámetro cuadrático del vuelo remanente $(\mathrm{cm})$. $d_{e}=$ diámetro cuadrático del vuelo extraído $(\mathrm{cm}) \cdot d_{a}=$ diámetro cuadrático del vuelo original $(\mathrm{cm}) . N_{e}=$ árboles extraídos en el aclareo (árboles ha ${ }^{-1}$ ). $N_{d}=$ densidad del vuelo remanente (árboles ha-1). $G$ = área basal del vuelo original $\left(\mathrm{m}^{2}\right.$ ha $\left.\mathrm{a}^{-1}\right) \cdot G_{e}=$ área basal del vuelo extraído $\left(\mathrm{m}^{2} \mathrm{ha}^{-1}\right) \cdot C_{c}=$ parámetro que representa la razón del diámetro de los árboles removidos respecto de los árboles remanentes. $\operatorname{Si} C_{c}=1, d_{e}=$ $d_{d}$, el aclareo es sistemático. $\mathrm{Si} C_{c}<1, d_{e}<d_{d}$ el aclareo es por lo bajo; si $C_{c}>1, d_{e}>d_{d}$ es un aclareo de los dominantes.

La mortalidad se representó en términos de la densidad (árboles ha ${ }^{-1}$ ), distinguiéndose una mortalidad denso-independiente que se debe a factores distintos a la competencia (ejemplo: sequías, plagas), de particular importancia durante las primeras fases de crecimiento; y una mortalidad denso-dependiente ocurrida por la competencia entre los individuos de la plantación (no se incorporó la competencia por malezas). Debido a que los cambios en la densidad del rodal también son afectados por la extracción de árboles mediante aclareos, que es un evento instantáneo y por eventos con un componente aleatorio (mortalidad denso-independiente); en lugar de modelar este componente como una ecuación diferencial, se modeló como una función en partes, donde la mortalidad denso-dependiente se describe con una curva trazada a mano alzada en un gráfico mediante la opción Sketch Graph de SIMILE que calcula el número de árboles que mueren en el rodal cada año en el que $G$ esté entre 15 y $37,5 \mathrm{~m}^{2} \mathrm{ha}^{-1}$ (figura 2). La mortalidad denso-independiente, se calculó para rodales entre cero y tres años mediante una función aleatoria que depende de la edad [10]:

$$
M_{d i}=\frac{n}{e d a d}
$$

Donde:

$M_{d i}=$ número de árboles muertos (árboles ha ${ }^{-1}$ año $\left.0^{-1}\right) \cdot n=$ número aleatorio entre 50 y 100 . edad $=$ edad de la plantación (años), edad $=0,1,2,3$. Esta parte de la función deja de operar después de los tres años de edad.

Finalmente, de [9] se obtiene el número de árboles extraídos en los aclareos. La densidad del rodal $N_{t+1}$ (árboles $\left.\mathrm{ha}^{-1}\right)$ viene dada por [11]:

$$
N_{t+1}=N_{t}-\left(M_{d i}+M_{d d}+M_{a}\right)_{t}
$$


Donde:

$N_{t}=$ densidad en el tiempo $\mathrm{t}$ (árboles ha ${ }^{-1}$ ). $M_{d i}=$ número de árboles muertos (árboles ha-1 año-1) por mortalidad denso-independiente. $M_{d d}=$ número de árboles muertos (árboles ha ${ }^{-1}$ año ${ }^{-1}$ ) por mortalidad denso-dependiente. $M_{a}$ $=$ número de árboles extraídos en el aclareo (árboles ha ${ }^{-1}$ año-1). Igual que $N_{e}$ en [9].

El volumen maderable se calculó como el volumen con corteza $\left(V_{c c}\right)$ y sin corteza $\left(V_{s c}\right)$ en $\mathrm{m}^{3}$ hasta $5 \mathrm{~cm}$ de diámetro en el extremo menor del árbol para el árbol promedio (ecuaciones [12] y [13] y el volumen total para el rodal mediante las ecuaciones de Moret et al. (1998):

$$
\begin{gathered}
V_{c c}=\beta_{6}\left(d^{2} \cdot h_{t}\right)^{\beta_{7}} \\
V_{s c}=\beta_{8}\left(d^{2} \cdot h_{t}\right)^{\beta_{9}}
\end{gathered}
$$

Donde,

$d=$ diámetro a la altura de pecho sobre corteza $(\mathrm{m}) . h_{t}=$ altura total promedio $(\mathrm{m}) . \beta_{6,9}$, son parámetros.

La cantidad de carbono almacenada cada año en el rodal se calculó a partir del volumen total con corteza en pie. Se consideró tanto la biomasa aérea como la biomasa subterránea (Quintero 2013) y se utilizaron los valores de conversión y expansión de biomasa aérea y subterránea determinados por Kraenzel et al. (2003) para plantaciones de teca. El carbono fijado como biomasa aérea en el año $i$ $\left(C_{\text {bai }}\right)$ viene dado por [14]:

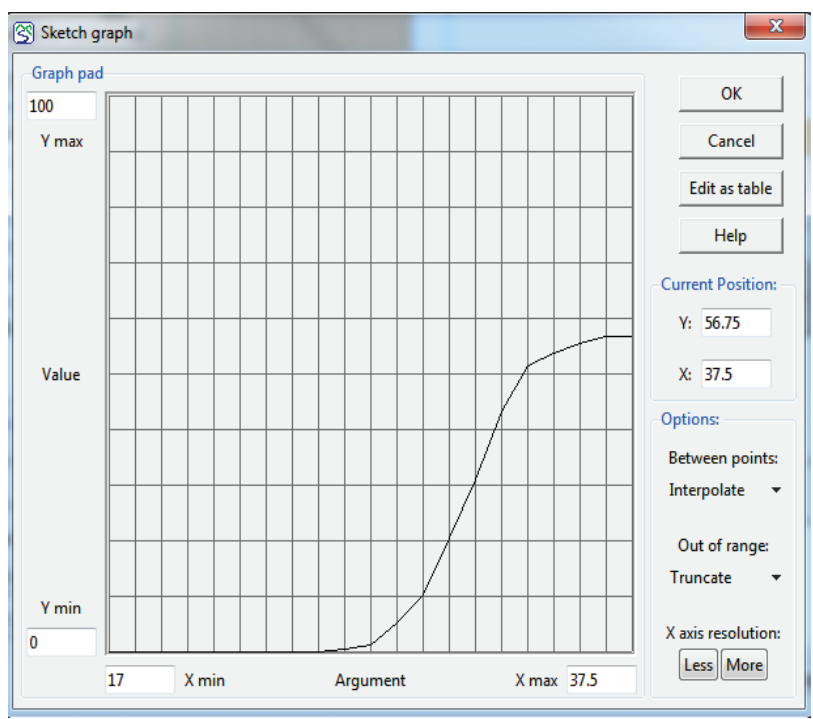

Figura 2. Representación de la mortalidad denso-dependiente en función del área basal del rodal mediante una curva a mano alzada.

Representing the density-dependent mortality of a stand as a function of basal area through a hand-free drawn curve.

$$
C_{b a i}=\Delta V_{i} \cdot d_{t e c a} \cdot f e b \cdot \gamma
$$

Donde, $\Delta V i=$ incremento corriente anual en volumen $\left(\mathrm{m}^{3} \mathrm{ha}^{-1}\right) \cdot d_{\text {teca }}=$ densidad básica de la teca $\left(\mathrm{Mg} \mathrm{m}^{-3}\right) \cdot \mathrm{feb}=$ factor de expansión para la biomasa aérea. $\gamma=$ proporción de carbono en la biomasa seca.

El carbono fijado en la biomasa subterránea en el año i $\left(C_{b s i}\right)$ viene dado por [15]:

$$
C_{b s i}=r \cdot C_{b a i}
$$

Donde $\mathrm{r}=$ relación raíz-vástago.

Construcción del modelo. Los datos se obtuvieron de plantaciones de teca establecidas en los Llanos Occidentales de Venezuela, incluyendo información de parcelas permanentes de aclareo y rendimiento, así como parcelas temporales que abarcan desde los dos hasta los 30 años de edad, con espaciamientos iniciales de entre 2,0 x 2,0 y 4,0 x 4,0 m. Se incluyeron plantaciones con cero a cuatro aclareos realizados a diferentes edades y con intensidad variable (cuadro 1).

Parametrización del modelo: los parámetros de crecimiento y rendimiento del modelo fueron estimados a partir de los datos de campo mediante regresión lineal y no lineal, empleando los procedimientos REG y NLIN de SAS v.9.1 (SAS Institute 2004). Los restantes parámetros fueron obtenidos de diferentes estudios (cuadro 2).

Implementación del modelo. El diagrama del modelo, dibujado en la interfaz gráfica de SIMILE, (figura 3) representa un rodal de plantación y consta de cinco submodelos: crecimiento, volumen en pie, volumen extraído, volumen muerto y carbono almacenado. La interfaz permitió diseñar el modelo, representando las variables y sus interrelaciones. Al introducir las correspondientes relaciones matemáticas, el modelo generó el código ejecutable y la documentación del programa. En SIMILE, la figura 3 no es únicamente un diagrama, sino el propio simulador el cual permite correr las simulaciones para observar el comportamiento del sistema y evaluar diferentes escenarios de manejo.

Para un conjunto de condiciones iniciales, la evolución del sistema fue simulada a través de integración numérica (se usó el método de Runge-Kutta).

En las condiciones iniciales se utilizó $t_{0}=0$, equivalente al momento de la implantación. Se asumió que $G$ inicial para cada densidad viene dado por la densidad inicial, multiplicado por el diámetro en el cuello de la raíz para los plantones ( $1 \mathrm{~cm}$ en promedio); y $H$ inicial para la curva guía $=0,5 \mathrm{~m}$. El periodo de simulación fue de 30 años desde la implantación hasta la corta final.

Se probaron 16 escenarios de manejo (E) en términos de regímenes de espesura (combinaciones de espaciamiento inicial, frecuencia, edad e intensidad de los aclareos) y calidad de sitio. Las calidades de sitio fueron (1) CS-I $=24 \mathrm{~m}$, 
Cuadro 1. Datos utilizados para la construcción del modelo. Plantaciones de T. grandis en los Llanos Occidentales de Venezuela. Data used for model construction. Tectona grandis plantations in the Western Plains of Venezuela.

\begin{tabular}{ccccccc}
\hline Localidad & $\begin{array}{c}\text { Tipo de } \\
\text { parcela }\end{array}$ & $\begin{array}{c}\mathrm{N}^{\circ} \text { de } \\
\text { parcelas }\end{array}$ & $\begin{array}{c}\text { Tamaño } \\
\text { parcela }\left(\mathrm{m}^{2}\right)\end{array}$ & $\begin{array}{c}\text { Densidad } \\
\left(\text { árboles ha }{ }^{-1}\right)\end{array}$ & $\begin{array}{c}\text { Edad } \\
(\text { años })\end{array}$ & $\begin{array}{c}N^{\circ} \\
\text { mediciones }\end{array}$ \\
\hline R.F. Caparo U.E: & $\mathrm{P}$ & 28 & $600-1600$ & $300-2.500$ & 2 a 30 & $25-32$ \\
\hline R.F. Caparo (IMADELLCA) & $\mathrm{T}$ & 57 & 280 & $500-1.200$ & $12-13$ & 2 \\
\hline R.F. Ticoporo (EMALLCA) & $\mathrm{T}$ & 44 & 1000 & $700-1.200$ & 8 & 2 \\
\hline R.F. Ticoporo & $\mathrm{T}$ & 514 & 250 & $300-1.200$ & $17-24$ & 2 \\
& $\mathrm{~T}$ & 88 & 800 & $500-1.000$ & $5,7-9$ & 2 \\
\hline AGROVENCA & $\mathrm{P}$ & 30 & 250 & $300-1.200$ & $17-19$ & 2 \\
\hline
\end{tabular}

$* \mathrm{P}=$ parcelas permanentes, $\mathrm{T}=$ parcelas temporales; R.F. $=$ reserva forestal, U.E. $=$ Unidad Experimental

Cuadro 2. Parámetros estimados a partir de diferentes estudios para plantaciones de $T$. grandis (todos los coeficientes estimados fueron significativos, $P<0,001$ ).

Parameter estimates from various studies for $T$. grandis plantations (all coefficients were significant, $P<0.001$ )

\begin{tabular}{|c|c|c|c|c|}
\hline Submodelo & Ecuación & Parámetro & Estimado & Referencia \\
\hline \multirow{3}{*}{ Altura mayor (m) } & 3 & $b_{1}$ & 1,42417 & \multirow{3}{*}{ Quintero et al. (2012) } \\
\hline & & $b_{2}$ & 0,45428 & \\
\hline & & $b_{3}$ & 0,23630 & \\
\hline Altura promedio (m) & 4 & $\delta$ & 0,93400 & Quintero et al. (2012) \\
\hline \multirow{3}{*}{ Área basal $\left(\mathrm{m}^{2} \mathrm{ha}^{-1}\right)$} & $5,6,7$ & $G_{p s}$ & 37,50000 & \multirow{3}{*}{ Zambrano et al. (1995) } \\
\hline & & $b_{4}$ & 0,08338 & \\
\hline & & $b_{5}$ & 0,91008 & \\
\hline \multicolumn{5}{|l|}{ Volumen total $\left(\mathrm{m}^{3} \mathrm{ha}^{-1}\right)$} \\
\hline \multirow[t]{2}{*}{ Con corteza } & 12 & $b_{6}$ & 0,34338 & \multirow{4}{*}{ Moret et al. (1998) } \\
\hline & & $b_{7}$ & 0,93383 & \\
\hline \multirow{2}{*}{ Sin corteza } & 13 & $b_{8}$ & 0,26006 & \\
\hline & & $b_{9}$ & 0,96364 & \\
\hline \multirow{4}{*}{$\begin{array}{l}\text { Secuestro de carbono } \\
\left(\mathrm{Mg} \mathrm{ha}^{-1}\right)\end{array}$} & 14,15 & $D_{\text {teca }}$ & 0,54000 & \multirow{4}{*}{$\begin{array}{l}\text { Pérez y Kanninen (2005); } \\
\text { Kraenzel et al. (2003) }\end{array}$} \\
\hline & & $\mathrm{Feb}$ & 1,33000 & \\
\hline & & $\gamma$ & 0,49500 & \\
\hline & & $r$ & 0,16000 & \\
\hline
\end{tabular}




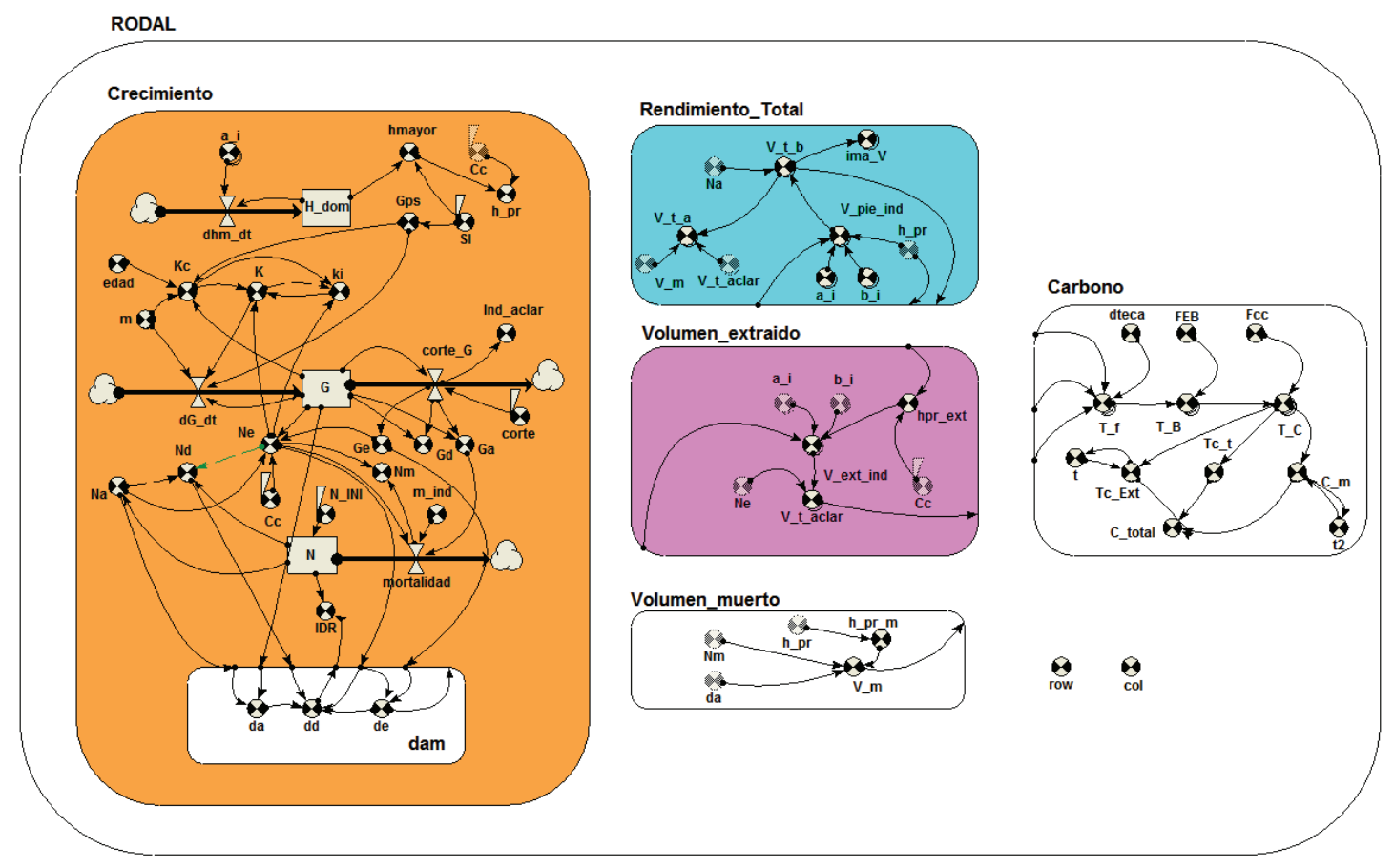

Figura 3. Diagrama-simulador del modelo de crecimiento y rendimiento para plantaciones de $T$. grandis en los Llanos Occidentales de Venezuela.

Diagram simulator of the growth and yield model for T. grandis plantations in the Western Plains of Venezuela.

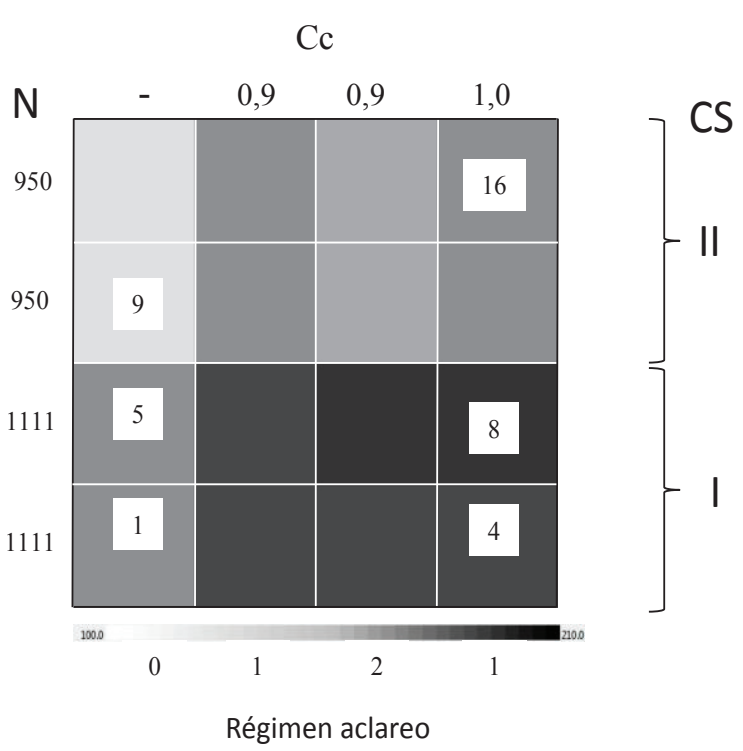

Figura 4. Representación espacial de los 16 escenarios en una grilla de 4 x 4. $\mathrm{CS}=$ Calidad de sitio I y II; $\mathrm{N}=$ densidad inicial (1111 y 950 árboles ha ${ }^{-1}$ ); régimen de aclareo (0: sin aclareos; 1 : un aclareo; 2: dos aclareos); y $C_{c}=$ clase de aclareo (-- no aclareo; 0,9: aclareo por lo bajo; 1,0: aclareo sistemático).

Spatial representation of 16 scenarios in a $4 \times 4$ grid. CS $=$ site quality I and II; $\mathrm{N}=$ initial stand density $\left(1,111\right.$ and 950 trees ha- $\left.{ }^{-1}\right)$; thinning schedule (0: no thinning; 1: one thinning; 2: two thinnings); and $\mathrm{Cc}=$ thinning class (--: no thinning; 0.9: low thinning; 1.0 : systematic thinning).
$G=37,5 \mathrm{~m}^{2} \mathrm{ha}^{-1}$; (2) CS-II $=21 \mathrm{~m}, G=34 \mathrm{~m}^{2} \mathrm{ha}^{-1}$; las densidades iniciales fueron (1) 1.111 árboles ha-1 ; (2) 950 árboles ha-1 ${ }^{-1}$ las clases de aclareo fueron (0) $C_{\mathrm{c}}=0,9$ aclareo por lo bajo de mediana intensidad y (1) $C_{\mathrm{c}}=1$ aclareo sistemático; el número de aclareos fue (0) sin aclareos; (1) aclareo automático en el que se extraen $8 \mathrm{~m}^{2} \mathrm{ha}^{-1}$ de área basal cada vez que el rodal alcanza $25 \mathrm{~m}^{2} \mathrm{ha}^{-1}$; (2) un aclareo no comercial a los 3 años de edad y un último aclareo a los 12 años, extrayéndose en ambos casos un $50 \%$ del área basal. Estos escenarios cubren una combinación de opciones de manejo que se han utilizado en plantaciones de teca. Por ejemplo, el espaciamiento inicial tradicional en teca ha sido de 3,0 $\mathrm{m} \mathrm{x} \mathrm{3,0} \mathrm{m} \mathrm{(1.111} \mathrm{árboles} \mathrm{ha-1)} \mathrm{con} \mathrm{varias} \mathrm{interven-}$ ciones moderadas a lo largo del turno; pero hoy día tienden a favorecerse regímenes con espaciamientos más amplios y menos aclareos, pero más intensos a edades tempranas (Ladrach 2009). La clase de aclareo en teca es usualmente por lo bajo, aunque con tendencia a métodos sistemáticos.

En SIMILE, teniendo las coordenadas reales de los rodales es posible realizar fácilmente la visualización espacial de las simulaciones, pudiéndose representar rodales de diferentes superficies y formas. En este caso, sólo se representaron rodales cuadrados adyacentes de una hectárea de superficie en una grilla de 4 x 4 donde cada celda corresponde a un escenario (figura 4). Por ejemplo, el escenario 9 corresponde a un rodal creciendo en calidad de sitio II con espaciamiento inicial de 950 árboles ha-1 donde no se efectúan aclareos a lo largo del turno. 


\section{RESULTADOS}

Para los escenarios E1-E4 (calidad de sitio 1: $\mathrm{N}=1.111$ árboles ha ${ }^{-1}$; regímenes 0,1 y 2 y $C_{c}=0,9$ aclareo por lo bajo y $C_{c}=1$ aclareo sistemático) se graficó el crecimiento en área basal, densidad, diámetro cuadrático y volumen sin corteza entre 0 y 30 años (figura 5). El crecimiento en área basal, para el escenario E1 (sin aclareos) se alcanzó rápidamente el $G_{p s}\left(37,5 \mathrm{~m}^{2}\right.$ ha-1 para CS-I) (figura $\left.5 \mathrm{~A}\right)$. Para los escenarios E2 y E4 (aclareos automáticos), el área basal se comportó de la misma manera (las líneas se traslapan) y se mantuvo dentro de una banda de 15 a $25 \mathrm{~m}^{2}$ $\mathrm{ha}^{-1}$, mientras que en el escenario E3 con sólo dos aclareos el área basal estuvo cercana al área basal potencial del sitio para el momento del turno. En cuanto a la densidad, todos los escenarios mostraron una reducción por mortalidad denso-independiente durante los tres primeros años.
En el escenario E1 al no realizarse aclareos la densidad se estabilizó, pero comenzó a reducirse después de alcanzar los $15 \mathrm{~m}^{2} \mathrm{ha}^{-1}$ debido a mortalidad denso-dependiente. En los demás escenarios, la mortalidad denso-dependiente fue muy baja, debido a la extracción de árboles en los aclareos (figura 5B). En el escenario E1, para la edad de corta, el diámetro cuadrático se mantuvo relativamente bajo (aprox. $23 \mathrm{~cm}$ ), mientras que para el E3 con un aclareo muy temprano, se obtuvo el mayor diámetro $(\cong 45 \mathrm{~cm})$. Los escenarios con régimen automático (E2 y E4) fueron muy similares, aunque se observó mayor crecimiento diamétrico con el aclareo sistemático (figura 5C). Nótense los saltos instantáneos en el crecimiento diamétrico consecuencia de la eliminación de árboles durante el aclareo. Finalmente, el volumen en pie sin corteza para el momento de la cosecha final (figura 5D) estuvo entre 150 y $350 \mathrm{~m}^{3}$ $\mathrm{ha}^{-1}$ siendo máximo cuando no se realizaron aclareos y mí-
A

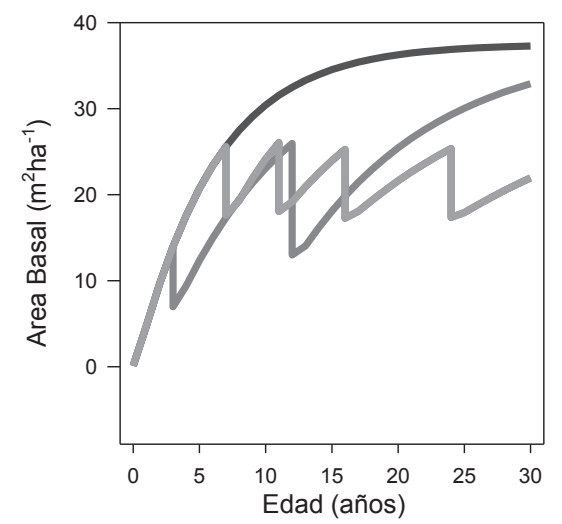

C

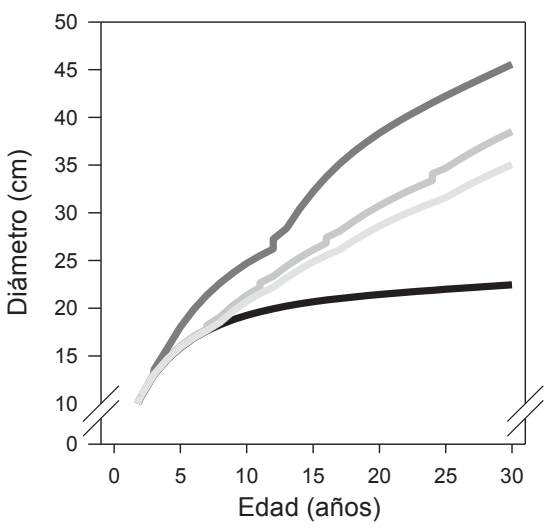

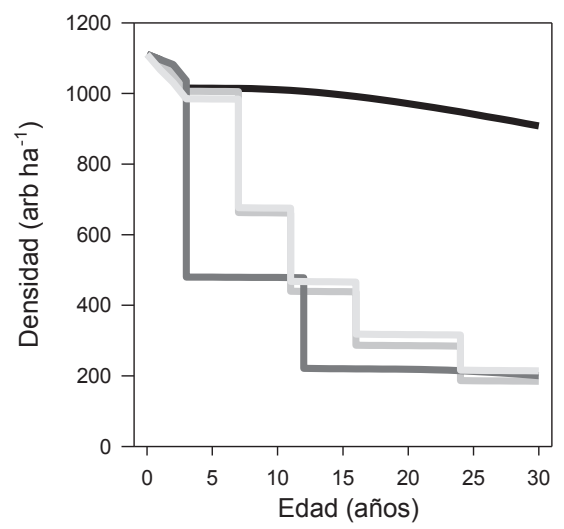

D

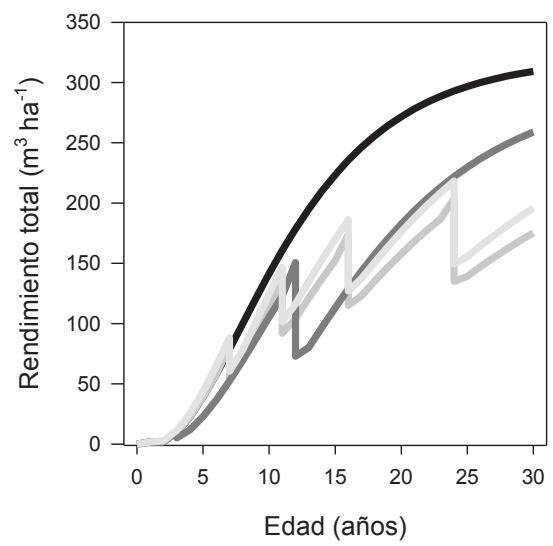

Figura 5. Salidas principales del simulador para para los escenarios E1 a E4. A) Área basal $\left.\left(\mathrm{m}^{2} \mathrm{ha}^{-1}\right), \mathrm{B}\right)$ densidad (árboles ha $\left.{ }^{-1}\right)$, C) diámetro cuadrático $(\mathrm{cm}), \mathrm{D})$ rendimiento volumétrico total $\left(\mathrm{m}^{3} \mathrm{ha}^{-1}\right)$. Color de líneas: E1 Sin aclareos ( $)$; E2 Aclareo automático moderado ( $\square$ ); E3 Aclareo intenso (-); E4 Aclareo automático sistemático ( $\square)$.

Main outputs for scenarios E1-E4. A) Basal area $\left(\mathrm{m}^{2} \mathrm{ha}^{-1}\right)$; B) stand density (trees ha-1); C) quadratic diameter (cm); D) total overbark volume $\left(\mathrm{m}^{3} \mathrm{ha}^{-1}\right)$. Line color: E1 no thinning (-); E2 moderate automatic thinning ( -$)$; E3 intensive thinning ( - ); E4 systematic thinning $(-)$. 
nimo con el aclareo automático sistemático. Sin embargo, el volumen total producido (cosecha + aclareos) fue mayor al realizar aclareos automáticos, donde casi la mitad de la madera producida correspondió a los aclareos (figura 6).

El escenario E2 con aclareo temprano tuvo menor producción total en volumen; sin embargo, debe tomarse en cuenta que el diámetro promedio fue considerablemente mayor que en los demás. La teca es una especie destinada principalmente a la producción de madera aserrada y las trozas de gran diámetro suelen tener un precio muy superior al de las trozas de pequeñas dimensiones. Por otra parte, cuando no se hicieron aclareos o un solo aclareo, la pérdida en volumen por árboles muertos fue mayor.

El carbono total secuestrado que incluye la biomasa de la cosecha final, los aclareos y los árboles muertos durante el crecimiento de la plantación para los 16 escenarios es representado espacialmente (figura 7). El secuestro de carbono estuvo entre 120 y $190 \mathrm{Mg} \mathrm{ha}^{-1}$, siendo más elevado en los escenarios de calidad de sitio I y régimen de aclareo automático sistemático (escenarios 4 y 8 ) con $188 \mathrm{Mg} \mathrm{ha}^{-1}$ en promedio. El menor secuestro (123 $\left.\mathrm{Mg} \mathrm{ha}^{-1}\right)$ ocurrió en los escenarios de CS-II sin aclareos (escenarios 9 y 13). La calidad de sitio fue el factor que más afectó el secuestro de carbono en promedio (CS-I $=171 \mathrm{Mg} \mathrm{ha}^{-1}$; CS-II $=138 \mathrm{Mg}$ $\left.\mathrm{ha}^{-1}\right)$. En segundo lugar, el régimen de aclareo, donde los escenarios de aclareo automático sistemático almacenaron $171 \mathrm{Mg} \mathrm{ha}^{-1}$ en promedio. Los escenarios con aclareo temprano presentaron menor secuestro de carbono que los escenarios con aclareo automático. Los escenarios sin aclareos almacenaron $140 \mathrm{Mg} \mathrm{ha}^{-1}$ en promedio. La diferencia en densidad inicial seleccionada no tuvo prácticamente influencia en las cantidades de carbono almacenadas.

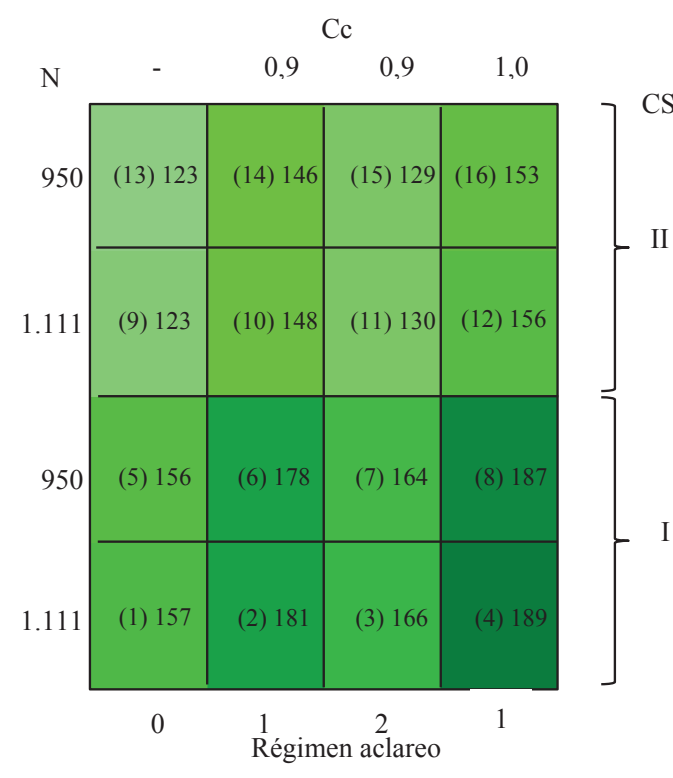

100

200

Figura 7. Carbono total almacenado $\left(\mathrm{Mg} \mathrm{ha}^{-1}\right)$ en las plantaciones de teca a los 30 años para los 16 escenarios representados en la grilla de 4 x 4 donde cada celda corresponde a un escenario. $\mathrm{CS}=$ Calidad de sitio I y II; $\mathrm{N}=$ densidad inicial (1.111 y 950 árboles ha ${ }^{-1}$ ); régimen de aclareo (0: sin aclareos; 1 : un aclareo; 2 : dos aclareos); y $\mathrm{Cc}=$ clase de aclareo (-- no aclareo; 0,9: aclareo por lo bajo; 1,0: aclareo sistemático).

Total carbon $\left(\mathrm{Mg} \mathrm{ha}^{-1}\right)$ stored in the teak plantations at age 30 for the 16 scenarios represented in the $4 \times 4$ grid. $C S=$ site quality I and II; $\mathrm{N}=$ initial stand density (1111 and 950 trees ha $\left.{ }^{-1}\right)$; thinning schedule (0: no thinning; 1: one thinning; 2 : two thinnings); and $\mathrm{Cc}=$ thinning class (--: no thinning; 0.9: low thinning; 1.0: systematic thinning).

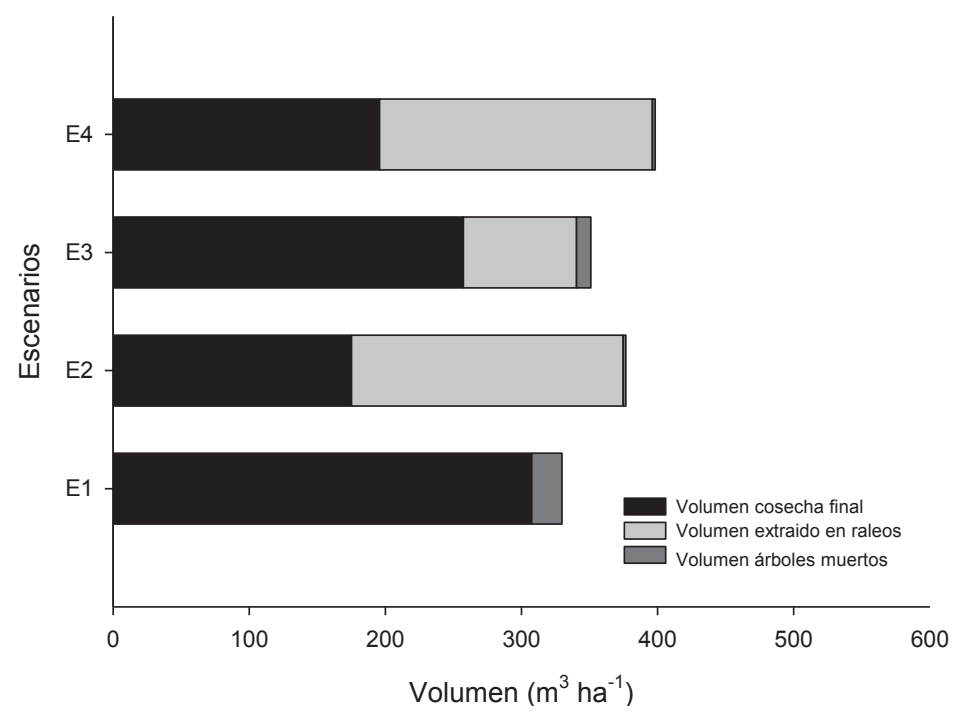

Figura 6. Volumen $\left(\mathrm{m}^{3} \mathrm{ha}^{-1}\right)$ de cosecha final, aclareos y árboles muertos para los escenarios: E1 Sin aclareos; E2 Aclareo automático moderado; E3 Aclareo intenso; E4 Aclareo automático sistemático.

Overbark volume $\left(\mathrm{m}^{3} \mathrm{ha}^{-1}\right)$ from the final harvest, from thinning and from dead trees for the scenarios: E1 no thinning; E2 moderate automatic thinning; E3 intensive thinning; E4 systematic automatic thinning. 


\section{DISCUSIÓN Y CONCLUSIONES}

La comparación de relaciones existentes entre las variables del rodal observadas en la literatura con las salidas del modelo, permiten detectar posibles deficiencias en los supuestos y ecuaciones utilizadas. Por ejemplo, el índice de densidad del rodal de Reineke que relaciona el diámetro cuadrático del rodal con la cantidad máxima de árboles que este puede soportar independientemente de la calidad de sitio y la edad de la plantación ha sido estimado entre 1050 y 1200 árboles por hectárea para la teca (Kumar et al. 1995, Jerez et al. 2003, Pérez y Kanninen 2005). El índice de densidad del rodal máximo generado por el modelo es de 900 árboles por hectárea para rodales sin aclarear, si bien es inferior a lo mencionado en la literatura, es razonable. La diferencia se debe a la curva utilizada para representar la mortalidad denso-dependiente como función del área basal solamente y a que no fue ajustada con una ecuación matemática. García et al. (2011) han propuesto ecuaciones que relacionan la mortalidad con densidad actual y la altura dominante del rodal, ya que estas variables presentan una mejor asociación causal con la mortalidad que el área basal que es más bien una expresión de la madera acumulada. En SIMILE, esta ecuación puede ser sustituida por otra más apropiada, sin necesidad de alterar el resto del modelo utilizando la capacidad Plug and Play del lenguaje.

Las salidas producidas por el modelo concuerdan con resultados obtenidos por estudios con información proveniente de parcelas permanentes y temporales en Venezuela y otras regiones de Latino América. Vincent et al. (2005) encontraron volúmenes con corteza de entre 170 y $380 \mathrm{~m}^{3}$ ha $^{-1}$ para plantaciones de 19 años de edad en la reserva forestal Caparo, correspondiendo los mayores volúmenes a las plantaciones sin aclarear, con alta densidad inicial (2.500 árboles $\mathrm{ha}^{-1}$ ) y en calidad de sitio I. Estas plantaciones alcanzaron un área basal potencial de sitio de $36,5 \mathrm{~m}^{3}$ ha ${ }^{-1}$, mientras que el diámetro cuadrático se mantuvo bajo $(17 \mathrm{~cm})$. Asimismo, los mayores diámetros se obtuvieron con las plantaciones establecidas a espaciamientos amplios (600 árboles ha-1) y aclareos intensos. Las plantaciones con varios aclareos moderados y espaciados presentaron volúmenes entre 200 y $300 \mathrm{~m}^{3} \mathrm{ha}^{-1}$ y diámetros entre 20 y $25 \mathrm{~cm}$. Asimismo, el volumen total con corteza para plantaciones de 24 años de edad en Costa Rica ha sido estimado entre 225 y $524 \mathrm{~m}^{3} \mathrm{ha}^{-1}$ correspondiendo los máximos valores a parcelas no aclareadas y de muy alta densidad (Pérez y Kanninen 2005). Estos autores también señalan que en turnos de 30 años es posible obtener diámetros entre 24,9 y $47,8 \mathrm{~cm}$; mientras que para rodales no aclareados, han encontrado un área basal potencial de sitio de hasta $40 \mathrm{~m}^{2} \mathrm{ha}^{-1}$. Bermejo et al. (2004) proyectaron volúmenes comerciales totales de entre 150 y $250 \mathrm{~m}^{3} \mathrm{ha}^{-1}$ para la teca a los 25 años a partir de información de plantaciones de entre 10 y 18 años de edad para una serie de sitios y regímenes de aclareo.
La relativa facilidad para correr múltiples escenarios, así como hacer modificaciones al modelo teniendo a la vista su estructura y los resultados de las corridas de manera simultánea facilitan mucho la experimentación y análisis de los resultados en SIMILE. Por ejemplo, se encontró que la calidad de sitio es el factor más importante en el almacenamiento de carbono en las plantaciones de teca, ya que este fue mayor en la calidad de sitio I, independientemente del espaciamiento inicial y los regímenes de aclareo en todos los escenarios (figura 6). La teca es muy exigente a condiciones climáticas (necesidad de un periodo de sequía) y de suelos (suelos bien drenados, fértiles y no excesivamente ácidos), lo que hace que la selección de sitios sea crítica para el éxito de las plantaciones con esta especie (Ladrach 2009).

No aclarear las plantaciones produce el máximo volumen en pie al alcanzarse el turno, aunque no necesariamente permite obtener el máximo volumen total si se toma en cuenta el volumen generado por los aclareos. Asimismo, al no ralear, los diámetros son más pequeños y la pérdida de volumen por mortalidad es más alta. Esto apunta claramente a la necesidad de hacer aclareos en las plantaciones de teca tomando en cuenta que el valor de los árboles aumenta de manera más que proporcional con el aumento en tamaño (Sage et al. 2013). El aclareo intenso y temprano y poco frecuente fue muy favorable para el crecimiento diamétrico, sin embargo, fue el que produjo menores volúmenes en pie para el turno y tampoco produjo el máximo volumen aprovechable durante los aclareos. Mientras que Ladrach (2009) propone aclareos tempranos e intensos en turnos de 20 a 25 años, Pérez y Kanninen (2005) proponen hasta cinco aclareos de intensidad variable en un turno de 30 años. De acuerdo con lo sugerido por Pérez y Kanninen (2005) y lo mostrado por el comportamiento del modelo, el incremento en área basal es capaz de mantenerse y alcanzar valores cercanos al potencial de sitio en respuesta a aclareos tardíos, por lo que en turnos relativamente largos ( 25 a 30 años), incluso con aclareos intensos, puede ser necesario realizar más de dos aclareos. La conveniencia de utilizar uno u otro régimen puede depender más de aspectos financieros y operativos que de aspectos biológicos. El análisis financiero para determinar el régimen más apropiado para las condiciones económicas y de mercado de diferentes regiones puede implementarse como un nuevo módulo sin afectar la estructura general del modelo. En general, los aclareos tempranos e intensos en turnos cortos serían más favorables en el contexto de altas tasas de interés y gran diferencial de precios según el tamaño de los árboles.

Los resultados del modelo para el almacenamiento total de carbono (aéreo y subterráneo a lo largo del turno) también son bastante razonables. Kraenzel et al. (2003) encontraron que plantaciones de teca madura, sin aclarear en Panamá, almacenaron en promedio $120 \mathrm{Mg} \mathrm{ha}^{-1}$ de carbono. Asimismo, Chanan e Iriany (2014) encontraron que el almacenamiento promedio para plantaciones de teca en Java es de aproximadamente $101 \mathrm{Mg} \mathrm{ha}^{-1}$ Estos estudios 
sólo consideran el carbono almacenado en el árbol en pie al momento de la cosecha. Quintero (2013) calculó los flujos de carbono y su almacenamiento en plantaciones de teca desde su establecimiento hasta el momento de retorno a la atmósfera del carbono almacenado en los diferentes productos generados por la plantación, encontrando entre 87 y $146 \mathrm{Mg} \mathrm{ha}^{-1}$.

Al usuario poco familiarizado con los conceptos de crecimiento y rendimiento de los rodales o con las implicaciones de las decisiones de manejo sobre el almacenamiento de carbono, la experimentación con el simulador le permite analizar de manera numérica y espacial el efecto de las decisiones de manejo representadas por cada escenario.

El modelado visual declarativo permite diseñar y experimentar con modelos de relativa complejidad de forma mucho más fácil, rápida y transparente que con los lenguajes de programación clásicos. El presente modelo, basado en el enfoque dinámico de espacio de estados es de utilidad para ilustrar los principales elementos en relación al crecimiento y manejo de una plantación forestal con fines de producción de madera y secuestro de carbono. Los ambientes de modelado visual declarativos basados en dinámica de sistemas pueden ayudar a desarrollar modelos de crecimiento de manera más intuitiva, al ofrecer una visión de conjunto que hace más evidente su estructura y supuestos críticos, haciendo mayor énfasis en la representación del sistema. No obstante, esto no libera al modelador de la necesidad de verificar la calidad del modelo mediante técnicas de validación que incluyen los análisis de sensibilidad, un riguroso examen de los supuestos utilizados y una comparación del comportamiento del modelo contra datos provenientes del sistema real (Vanclay y Skovsgaard 1997, Vanclay 2014).

Los ambientes de modelado visual como el SIMILE, proveen una serie de herramientas que permiten llevar a cabo estos análisis y comparaciones de manera mucho más eficiente que con lenguajes de programación de múltiple propósito usados todavía muy frecuentemente para el desarrollo de simuladores en el campo ecológico, forestal y agroforestal al facilitar la experimentación de alternativas que pueden abarcar desde la corrida simultánea de numerosos escenarios, hasta el análisis de la sensibilidad a cambios en los parámetros, lo cual puede ser muy útil en el examen de los supuestos del modelo, la calidad de los datos, el enfoque de construcción, la eficiencia de los algoritmos de computación y la calidad de las predicciones. La capacidad de dividir el modelo en módulos permite la implementación de diferentes sub-modelos para representar los procesos de crecimiento, rendimiento o agregar nuevas salidas sin necesidad de tener que recodificar todo el modelo.

\section{AGRADECIMIENTOS}

Este trabajo fue financiado por el Consejo de Desarrollo Científico, Humanístico, Tecnológico y de las Artes (CDCHTA), de la Universidad de Los Andes, Mérida, Venezuela, bajo el proyecto FO-686-08-01-B. Los autores quieren expresar su agradecimiento a tres revisores anónimos que contribuyeron a mejorar este manuscrito.

\section{REFERENCIAS}

Bermejo I, I Cañellas, A San Miguel. 2004. Growth and yield models for teak plantations in Costa Rica. Forest Ecology and Management 189: 97-110. doi: j.bbr.2011.03.031.

Chanan M, A Iriany. 2014. Estimating carbon storage on teak (Tectona grandis Linn. F). Journal of Environment and Earth Science 4: 9-17.

Forrester JW. 1961. Industrial Dynamics. Cambridge MA, USA. Massachusetts Institute of Technology Press. USA. 464 p.

García O. 1988. Growth modelling a (re)view. New Zealand Forestry 33(3): 14-17.

García O. 1994. The state-space approach in growth modelling. Canadian Journal of Forest Research 24(9): 1894-1903.

García O. 2013. Forest Stands as Dynamical Systems: An Introduction. Modern Applied Science 7: 32-38.

García O, H Burkhart, R Amateis. 2011. A biologically-consistent stand growth model for loblolly pine in the Piedmont physiographic region, USA. Forest Ecology and Management 262: 2035-2041.

Jerez M, L Vincent, A Moret, R González. 2003. Regímenes de espaciamiento inicial y aclareo en plantaciones de teca (Tectona grandis L.f.) en Venezuela. Memorias del seminario virtual en plantaciones de teca: posibilidades y perspectivas para su desarrollo (en CD). 26 al 28 de noviembre, Heredia, Costa Rica.

Kraenzel M, A Castillo, T Moore, C Potvin. 2003. Carbon storage of harvest-age teak (Tectona grandis) plantations, Panama. Forest Ecology and Management 173: 213-225.

Kumar B. J Long, P Kumar. 1995. A density management diagram for teak plantations of Kerala in peninsular India. $\mathrm{Fo}_{\mathrm{O}}$ rest Ecology and Management 74: 125-131.

Ladrach W. 2009. Manejo de plantaciones de la teca para productos sólidos. Sociedad Internacional de Forestales Tropicales (ISTF Noticias. Informe Especial). Consultado 15 ago. 2013. Disponible en http://www.istf-bethesda.org/specialreports/teca.pdf

Moret AY, M Jerez, A Mora. 1998. Determinación de ecuaciones de volumen para plantaciones de teca (Tectona grandis L.) en la Unidad Experimental de la Reserva Forestal Caparo, Estado Barinas-Venezuela. Revista Forestal Venezolana 42(1): 41-50.

Muetzelfeldt R, J Massheder. 2003. The SIMILE Visual Modelling Environment. European Journal of Agronomy 18(3): 345-358.

Muetzelfeldt R, J Taylor. 2001. Developing forest models in the Simile visual modelling environment. IUFRO 4.11 Conference on Forest Biometry, Modelling and Information Science. University of Greenwich, UK. Consultado 10 jul. 2013. Disponible en http://www.ierm.ed.ac.uk/simile/ documents/iufro3.pdf

Muetzelfeldt R, J Taylor. 2004. Getting to know Simile the visual modelling environment for ecological, biological and environmental research. Consultado 10 dic. 2013. Disponible en http://www.simulistics.com/home.html

Peng C. 2000. Growth and yield models for uneven-aged stands: past, present and future. Forest Ecology and Management 132(2-3): 259-279.

Pérez D, M Kanninen. 2005. Stand growth scenarios for Tectona 
grandis plantations in Costa Rica. Forest Ecology and Management 210: 425-441.

Pienaar LV, KJ Turnbull. 1973. The Chapman-Richards. Generalization of Von Bertalanffy's growth model for basal area growth and yield in even-aged stands. Forest Science 19: 2-22.

Quintero MA. 2013. Modelado de un sistema de planificación y manejo de plantaciones forestales industriales. Tesis Doctoral en Ciencias Aplicadas. Mérida, Venezuela. Facultad de Ingeniería, Universidad de Los Andes. 263 p.

Quintero MA, M Jerez, J Flores. 2012. Modelo de crecimiento y rendimiento para plantaciones de teca (Tectona grandis L.) usando el enfoque de espacio de estados. Ciencia e Ingeniería 33: 33-42.

Sage LF, J Kent, JP Morales. 2013. Rentabilidad de las inversiones de teca. In De Camino R, JP Morales eds. Las plantaciones de teca en América Latina: Mitos y realidades. Turrialba, Costa Rica. CATIE. p. 202-225.

SAS Institute Inc. 2014. Base SAS ${ }^{2} 9.1$ Procedures Guide. Cary, NC.
Vanclay JK. 2003. The one-minute modeller: an introduction to Simile. Annals of Tropical Research 25: 31-44.

Vanclay JK. 2014. Unsuspected implications arising from assumptions in simulations: Insights from recasting a forest growth model in system dynamics. Forest Ecosystems 1: 7. doi:10.1186/2197-5620-1-7.

Vanclay JK, JP Skovsgaard. 1997. Evaluating forest growth models. Ecological Modelling 98: 1-12.

Vincent L, A Moret, M Jerez. 2000. Comparación de algunos regímenes de espesura en plantaciones de teca en el Área Experimental de la Reserva Forestal de Caparo, Venezuela. Revista Forestal Venezolana 44(2): 87-95.

Weiskittel A, D Hahn, JA Kershaw, JK Vanclay. 2011. Forest Growth and Yield Modeling. Chichester, UK. Wiley-Blackwell. 415 p.

Zambrano T, M Jerez, L Vincent. 1995. Modelo preliminar de simulación del crecimiento en área basal para la teca (Tectona grandis L.) en los Llanos Occidentales de Venezuela. Revista Forestal Venezolana 39: 40-48. 